\title{
Société Suisse de Pathologie
}

Pierre-André Diener, Secrétaire de la SSPath

Dr Pierre-André Diene Institut de pathologie Hôpital cantonal Saint-Gall CH-9007 Saint-Gall p.a.diener@kssg.ch www.sgpath.ch

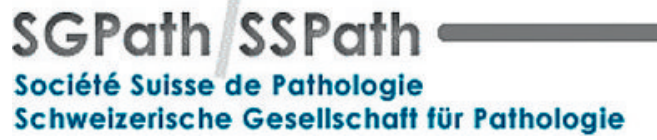

\section{Définition de la pathologie}

La pathologie consiste à comprendre les maladies, leurs origines, les processus en cours dans l'évolution de la maladie ainsi que les altérations morphologiques et moléculo-biologiques qui en résultent.

Les pathologistes sont peu connus du grand public car ils ne rencontrent que rarement les patients. Plus de $60 \%$ de la population croit qu'ils examinent seulement les personnes décédées. Or la pathologie est l'une des rares disciplines complètes diagnostiquant l'être humain de la tête aux pieds et secondant les autres disciplines pour déterminer et traiter les maladies. Les pathologistes accompagnent ainsi en arrière-plan les maladies de personnes vivantes. Les données saisies dans ce contexte sont indispensables à l'épidémiologie (p.ex. registre des cancers). Il est aussi important que le nombre d'autopsies pratiquées soit élevé pour la statistique des causes de décès, car il pourrait jouer un rôle important dans le contrôle de la qualité médicale.

\section{Activité en pathologie clinique}

Dans la médecine moderne, les examens de cellules et de tissus prélevés sur des personnes vivantes constituent plus de $90 \%$ du travail des pathologistes. Ceuxci détectent les maladies inflammatoires et les classent en fonction de leur étiologie. Presque toutes les tumeurs sont diagnostiquées dans le cadre de la pathologie. Lors de tumeurs malignes, les pathologistes procèdent à la saisie de paramètres importants pour la thérapie: typisation d'après la nomenclature de l'OMS, stade tumoral, aspect complet de la résection chirurgicale, degré de réponse de la chimiothérapie et/ou radiothérapie néo-adjuvante sur les pièces de résection chirurgicale.

Pour le traitement des tumeurs malignes, la détection des facteurs prédictifs déterminant la prescription de médicaments gagne en importance, telles la protéine Her2/antigène Her2 lors de carcinomes mammaires (Herceptin) ou la molécule CD20 dans les lymphomes à cellules B (Rituximab). Les examens préventifs deviennent toujours plus importants dans la détection précoce du cancer et la thérapie des lésions précancéreuses. On mentionnera ici la colonoscopie préventive et le screening du cancer du sein.

\section{Objectifs et champs d'activité de la SSPath}

La Société suisse de pathologie a été créée en 1935 à Berne. Elle compte actuellement 368 membres. La grande majorité travaille dans le domaine de la pathologie humaine, un tiers est engagé dans la recherche clinique. Des groupes plus petits exercent leur activité dans la pathologie vétérinaire, dans la recherche fondamentale / industrie pharmaceutique, ou dans des disciplines cliniques. La SSPath ainsi que ses groupes de spécialistes en cytopathologie et pathologie moléculaire poursuivent les objectifs suivants:

- promouvoir l'enseignement, les prestations et la recherche de la pathologie et de ses domaines spécialisés.

- établir des contacts avec les associations nationales et internationales de pathologie.

- réaliser la formation postgraduée et continue.

- promouvoir l'assurance de qualité.

- encourager la formation et le perfectionnement du personnel technique.

La SSPath constitue simultanément la section suisse de l'Académie Internationale de Pathologie. Elle organise chaque année un congrès et deux histoséminaires. Les groupes de travail de la SSPath se consacrent spécialement à la formation postgraduée et continue en pathologie des différents organes et systèmes d'organes. Ils forment le pool de spécialistes pour les questions spécifiques à la discipline et pour l'élaboration de directives concernant l'assurance de qualité.

\section{Problèmes et perspectives}

Dans le cadre de la réforme de Bologne, la pathologie est de plus en plus intégrée dans les cours où des représentants d'autres disciplines pourraient éventuellement l'enseigner. Si la pathologie n'est plus enseignée comme discipline de base, les conséquences à long terme seront les suivantes: l'enseignement systématique des bases sera négligé; les futurs médecins ne disposeront plus de connaissances de base aussi étendues et la qualité de la médecine pourrait diminuer. La pathologie en particulier sera insuffisamment prise en compte en tant que profession à part entière. La pénurie actuelle de pathologistes s'aggravera alors que les exigences diagnostiques augmenteront en parallèle. 